\title{
Tactile vibration: Dynamics of psychophysical scaling method, test site, and contactor surface area
}

\author{
DANIEL HARRIS \\ Healthcare Rehabilitation Center, Austin, Texas \\ DONALD FUCCI \\ Ohio University, Athens, Ohio \\ and \\ LINDA PETROSINO \\ Bowling Green State University, Bowling Green, Ohio
}

\begin{abstract}
The purpose of this pilot study was to investigate possible interactive effects of psychophysical scaling method, body test site, and contactor surface area on vibrotactile sensation magnitude functions. The scaling methods of magnitude estimation and magnitude production were employed to obtain vibrotactile magnitude functions for the anterior dorsum of the tongue and the thenar eminence of the hand. Three contactor areas were employed, and 10 young adults $(M=19.8$ years of age) participated as subjects. The results suggested interactive influences between scaling method, test site vibrotactile threshold, and number of sensory units activated by the stimulus.
\end{abstract}

In 1961, Stevens reported a series of experiments conducted to investigate the relationship between stimulus intensity and subjective magnitude. Controlled vibration was one of the stimuli studied, with the fingertip and forearm as the body test sites (Stevens, 1959). Since then, a number of investigators have attempted to determine the effects of various scaling methods, body test sites, and skin contactor surface areas on vibrotactile magnitude functions. For example, the psychophysical scaling methods of magnitude estimation and magnitude production have been used to study vibrotactile magnitude functions for the fingertip, thenar eminence of the hand, volar forearm, and dorsal surface of the tongue (Fucci, Harris, McMath, \& Petrosino, 1986; Fucci, Harris, \& Petrosino, 1983, 1984; Fucci \& Petrosino, 1983; Verrillo \& Chamberlain, 1972; Verrillo, Fraioli, \& Smith, 1969). The results of these investigations have indicated that, with other parameters held constant, the method of magnitude estimation yields shallower magnitude functions than does the method of magnitude production, cutaneous areas of higher vibrotactile threshold yield steeper magnitude functions than do areas of lower threshold, and magnitude function exponent values are inversely related to contactor surface area.

The fact that psychophysical scaling method, test site threshold, and contactor area may individually influence results leads to questions concerning the possible interactive effects that these parameters may have on vibrotactile magnitude functions. Verrillo and Chamberlain (1972) reported that the exponent values of magnitude

Request reprints from Daniel Harris, Healthcare Rehabilitation Center, 1106 W. Dittmar Lane, Austin, TX 78745. functions depend on the number of sensory units activated as a function of test site neural density, contactor area, and contactor surround conditions. Fucci et al. (1986) suggested that vibrotactile magnitude scaling behavior may be influenced by the interaction between an internal “absolute" scaling mechanism (Zwislocki \& Goodman, 1980 ), the scaling method chosen, and the range of stimulus intensities. To date, however, no experiment has been conducted to investigate the possible interactions between psychophysical scaling method, body test site, and vibrotactile contactor surface area. This research would seem particularly warranted in that these three parameters must be established in any study involving vibrotactile sensation magnitudes. The purpose of the present pilot study was to conduct a preliminary investigation of the possible interaction of these three factors. The methods of magnitude estimation and magnitude production were employed for two test sites, the anterior lingual dorsum and thenar eminence of the hand, using three contactor areas.

\section{METHOD}

\section{Subjects}

Ten subjects, 5 men and 5 women, ranging in age from 19 to 21 years $(M=19.8)$, were randomly selected from an introductory course in hearing and speech sciences. All of the subjects had normal speech and hearing and reported no known sensory or motor impairments. None of the subjects were experienced in vibrotactile testing.

\section{Apparatus}

The vibrotactile instrumentation consisted of a stimulus unit and a measurement unit. The stimulus unit was composed of a sine wave generator, an experimenter-controlled variable attenuator, a subject-controlled variable attenuator, a rise/fall gate, two universal timers, an audio am- 
plifier, a power amplifier, and an electromagnetic minivibrator with a probe-contactor extension. The pulsed vibratory signal had a frequency of $250 \mathrm{~Hz}$ and a $50 \%$ duty cycle (on $600 \mathrm{msec}$ and off $600 \mathrm{msec}$ ), with a rise and decay time of $50 \mathrm{msec}$. The measurement unit included an accelerometer, a cathode follower, a microphone amplifier, and a voltmeter. A noise generator was used to present narrow band noise centered around $250 \mathrm{~Hz}$ at a $70-\mathrm{dB}$ hearing threshold level to prevent the subjects from hearing the vibratory stimulus. A detailed description of the vibrotactile equipment and procedures can be found in a review by Harris, Fucci, Petrosino, and Wallace (1986).

\section{Procedure}

The vibrotactile test sites employed were the midline section of the dorsum of the tongue and the thenar eminence of the right hand. Three different contactors with areas of $.128, .320$, and $1.30 \mathrm{~cm}^{2}$ were used at both test sites for all 10 subjects. For each contactor, there was a corresponding rigidly mounted plastic disk, with a hole that allowed a 1-mm gap between the contactor and the disk. Each subject was seated in an adjustable chair and positioned so that the structure being tested could be placed against the bottom of the plastic disk. The vibrator contactor extension was then positioned to make contact with the test structure through the hole in the disk. For each subject, the ascending method of limits was used to obtain vibrotactile thresholds for each contactor size on the two test structures. This procedure was accomplished so that suprathreshold stimulus intensities required for magnitude estimation could be established in sensation levels for each subject (Verrillo et al., 1969).

Subsequent to the threshold testing, the psychophysical scaling methods of magnitude estimation and magnitude production were used for all $\mathbf{1 0}$ subjects to obtain vibrotactile magnitude functions for both test sites. The order of presentation for the three contactor sizes was randomized between subjects across all test sessions. Each subject participated in four sessions. During the first session 5 subjects performed magnitude estimation for the lingual dorsum and 5 subjects performed magnitude estimation for the thenar eminence. During the second session the test sites were reversed for the two subject groups, and the magnitude estimation procedure was repeated. For each session the subject assigned numbers to a randomly presented series of eight stimulus intensities ranging from 6 to $40 \mathrm{~dB}$ sensation level $(6,10,16,20,26,30,36$, and $40 \mathrm{~dB} S L)$. The subject was instructed to "think of a number that matched the strength" of each vibrotactile stimulus intensity presented. Whole numbers, decimals, and fractions were indicated as permissible selections (Zwislocki \& Goodman, 1980). The subjects were encouraged to be spontaneous in selecting numbers and to judge each stimulus without reference to those previously presented. The geometric means of all subject responses for each of the eight stimulus intensities were taken as the mean magnitude estimation responses for each test structure and contactor size.

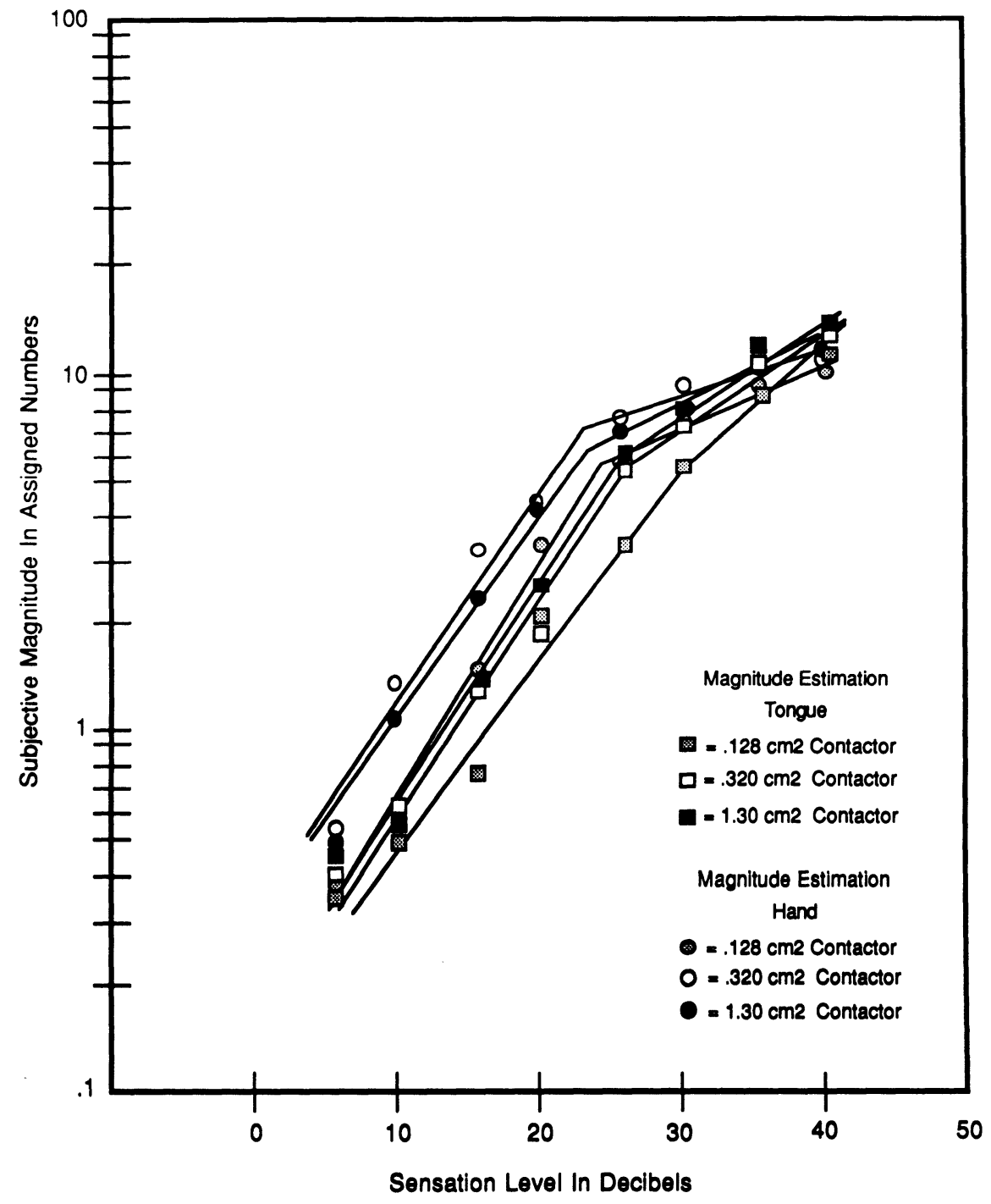

Figure 1. Vibrotactile magnitude estimation functions for the lingual dorsum and thenar eminence according to the three contactor areas employed. 
During the third test session 5 subjects performed magnitude production for the lingual dorsum and 5 subjects performed magnitude production for the thenar eminence. During the fourth session the test sites were reversed between the two subject groups, and the magnitude production procedure was repeated.

The stimulus numbers presented during the magnitude production task were derived from the geometric means of the numerical responses provided by the 10 subjects for the corresponding contactor size and test structure employed during the magnitude estimation task. For each contactor size and test structure, a single run of stimulus numbers was presented (Petrosino, Fucci, \& Harris, 1985). The subjects were instructed to adjust the intensity of the vibrotactile stimulus presented to the tongue or hand to match the magnitude of the number presented by the experimenter. The subject-controlled attenuator consisted of a smooth unmarked knob (120-dB potentiometer) that provided no visual or mechanical cues. The experimenter was in control of a master attenuator that could be used to vary the amplitude of the stimulus being delivered to the subject-controlled attenuator (Zwislocki \& Goodman, 1980). The data from the magnitude production task for all conditions were recorded in decibels sensation level. The geometric means of the vibrotactile stimulus intensity values provided by each of the 10 subjects for each contactor size on each test structure were taken as the magnitude production values for the subject group.

\section{RESULTS AND DISCUSSION}

Figure 1 shows the vibrotactile magnitude estimation functions obtained in the present experiment for the tongue and hand according to the three contactor sizes employed. Figure 2 shows the vibrotactile magnitude production functions for the same structures and contactor sizes.

The data in the figures suggest the possibility of interactive effects of psychophysical method, test site vibrotactile threshold, and contactor size on vibrotactile sensation magnitudes. For example, the lower slopes for lingual vibrotactile magnitude estimation and magnitude production were shallower for the $.128-\mathrm{cm}^{2}$ contactor than for the $.32-\mathrm{cm}^{2}$ and $1.3-\mathrm{cm}^{2}$ contactors. These data initially appeared to disagree with the hypothesis of an inverse relationship between contactor size and slope as proposed in previous research (Fucci et al., 1983; Verrillo \& Chamberlain, 1972); however, it may be that an interaction be-

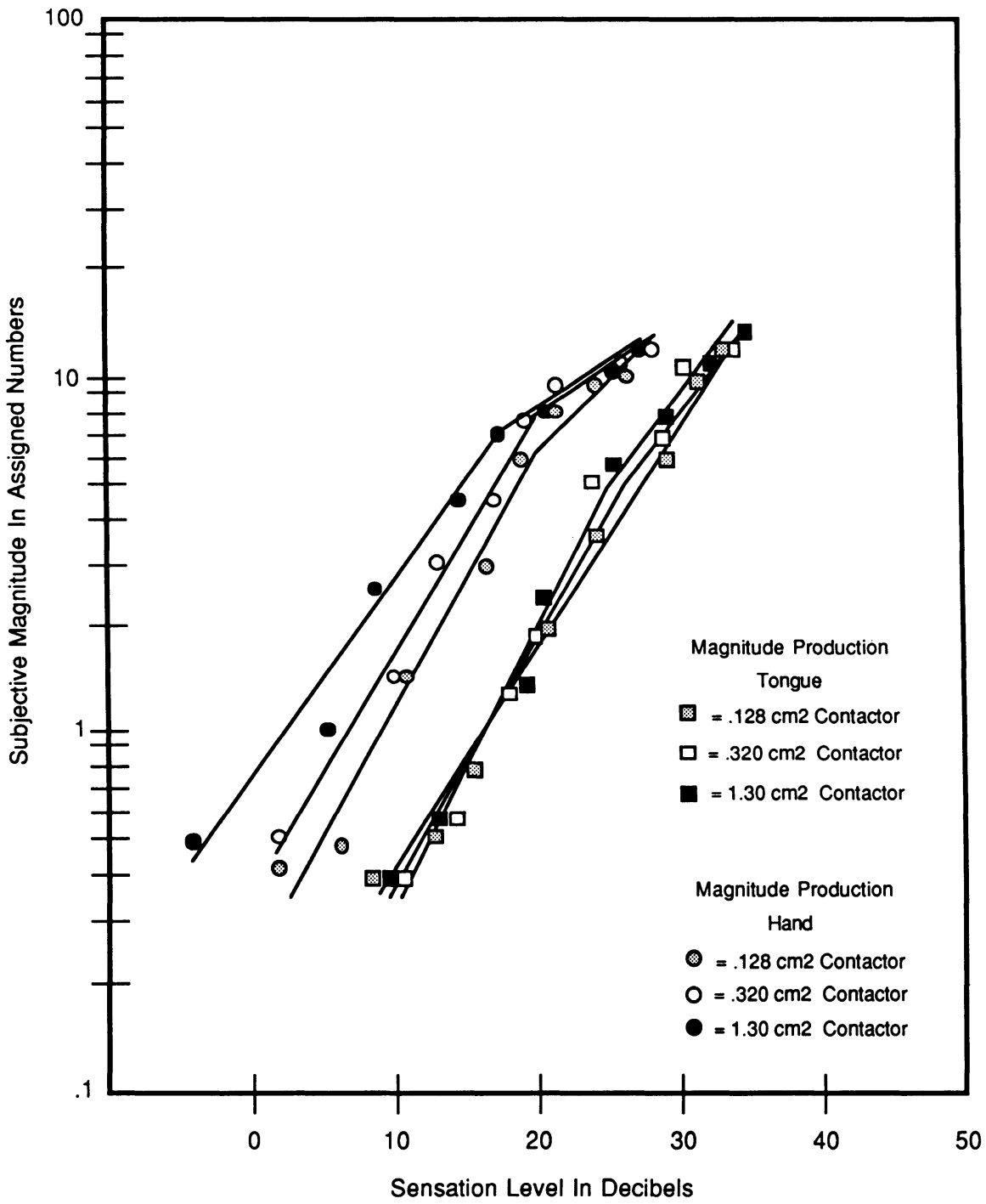

Figure 2. Vibrotactile magnitude production functions for the lingual dorsum and thenar eminence according to the three contactor areas employed. 
tween the lingual test site vibrotactile threshold and the small surface area of the $.128-\mathrm{cm}^{2}$ contactor influenced this result. Previous investigators have commented that changes in the slope of vibrotactile magnitude functions according to threshold conditions may be analogous to loudness recruitment (Békésy, 1955, 1958; Stevens, 1959; Verrillo \& Chamberlain, 1972); that is, conditions associated with higher thresholds may result in more rapid growth in sensation magnitude than conditions associated with lower thresholds. A typical range of thresholds for the lingual dorsum would be 1.0 to $2.5 \mathrm{~dB}$ re $1-\mu$ peak, whereas a typical range of thresholds for the thenar eminence would be -14.0 to $-10.0 \mathrm{~dB}$ re $1-\mu$ peak (Fucci \& Crary, 1979; Verrillo \& Chamberlain, 1972). The present lingual vibrotactile data for the $.128-\mathrm{cm}^{2}$ contactor may reflect recruitment-like sensation growth in that the magnitude estimation and magnitude production functions for the $.128-\mathrm{cm}^{2}$ contactor became steeper than the functions for the larger two contactors as stimulus intensity was increased. This result would be consistent with rapid sensation growth associated with interaction of a relatively high lingual threshold and a relatively small number of sensory units activated by the $.128-\mathrm{cm}^{2}$ contactor (Verrillo \& Chamberlain, 1972).

In addition, the fact that magnitude functions for the lingual dorsum showed a notably faster upper slope growth rate than those for the thenar eminence suggested that interaction of test site threshold and contactor area may have influenced the differences in slopes between the tongue and the hand. When compared with the threshold for the lingual dorsum, the relatively low threshold for the thenar eminence may have been associated with a slower growth in sensation magnitude at higher stimulus intensities across the three contactor sizes.

The possible interaction between vibrotactile test site threshold and contactor area in the present study was more apparent for the method of magnitude production than for the method of magnitude estimation. Figure 2 shows that lingual vibrotactile magnitude production functions for all three contactor sizes began at higher sensation levels and grew faster in upper slopes than magnitude production functions for the thenar eminence. This result suggested that when subjects were in control of the vibrotactile stimulus, their responses reflected neural conditions related to test site threshold and the number of sensory units activated (Békésy, 1955, 1958; Stevens, 1959; Verrillo \& Chamberlain, 1972). Zwislocki and Goodman (1980) investigated magnitude estimation and magnitude production scaling behavior for loudness and line length in several different subject groups. They concluded that subjects tend to produce magnitude estimation scales according to an internal "absolute" scaling mechanism whereby the same numerical units are used regardless of the location of stimulus range. Figure 1 of the present in- vestigation shows the relative similarity of vibrotactile magnitude estimation functions for the lingual dorsum and the thenar eminence. This data seemed to support the hypothesis of an absolute numerical scaling mechanism as proposed by Zwislocki and Goodman (1980). However, the differences between vibrotactile magnitude production functions for the lingual dorsum and thenar eminence, as shown in Figure 2, appeared to suggest that interaction of scaling method and the number of neural units activated may be a consideration in vibrotactile psychophysical experimentation.

Further research employing larger subject groups and statistical comparisons of the relationships between psychophysical scaling method, vibrotactile test site threshold, and contactor surface area appears to be warranted.

\section{REFERENCES}

BÉKÉSY, G. voN. (1955). Human skin perception of traveling waves similar to those on the cochlea. Journal of the Acoustical Society of America, 17, 830-841.

BéKÉSY, G. voN. (1958). Funneling in the nervous system and its role in loudness and sensation intensity on the skin. Journal of the Acoustical Society of America, 30, 399-412.

FuCCI, D., \& CRARY, M. (1979). Oral vibrotactile sensation and perception: State of the art. In N. Lass (Ed.), Speech and Language (Vol. 2, pp. 321-351). New York: Academic Press.

Fucci, D., Harris, D., McMath, E., \& Petrosino, L. (1986). Effects of exposure to psychophysical scaling on lingual vibrotactile magnitude estimation and magnitude production. Journal of Perceptual \& Motor Skills, 63, 67-74.

Fucci, D., Harris, D., \& Petrosino, L. (1983). The effect of contactor area on vibrotactile magnitude function exponents for the tongue and hand. Bulletin of the Psychonomic Society, 21, 400-402.

Fucci, D., Harris, D., \& Petrosino, L. (1984). Sensation magnitude scales for vibrotactile stimulation of the tongue and thenar eminence. Journal of Perceptual \& Motor Skills, 58, 843-848.

FucCI, D., \& Petrosino, L. (1983). Lingual vibrotactile sensation magnitudes: Comparison of suprathreshold responses for the tongue and hand. Journal of the Acoustical Society of America, 74, 351-353.

Harris, D., Fucci, D., Petrosino, L., \& Wallace, D. (1986). Instrumentation for magnitude estimation and cross-modality matching of auditory and lingual vibrotactile sensations. Review of Scientific Instruments, 57, 2343-2347.

Petrosino, L., Fucci, D., \& Harris, D. (1985). Effects of single session repetitive judgments on magnitude estimation scales for lingual vibrotactile sensation. Perception \& Psychophysics, 37, 205-208.

Stevens, S. S. (1959). Tactile vibration: Dynamics of sensory intensity. Journal of Experimental Psychology, 57, 210-218.

STEVENS, S. S. (1961). The psychophysics of sensory function. In W. A. Rosenblith (Ed.), Sensory Communication (pp. 1-33). New York: Wiley.

Verrillo, R. T., \& Chamberlain, S. C. (1972). The effect of neural density and contactor surround on vibrotactile sensation magnitude. Perception \& Psychophysics, 6, 366-372.

Verrillo, R. T., Fraioli, A. J., \& Smith, R. L. (1969). Sensation magnitude of vibrotactile stimuli. Perception \& Psychophysics, 6, 366-372.

ZWISLOCKI, J., \& GoOdMAN, D. (1980). Absolute scaling of sensory magnitudes: A validation. Perception \& Psychophysics, 28, 28-38.

(Manuscript received for publication February 19, 1988.) 